\title{
¿UN CURRÍCULUM ACERCA DEL JUEGO LIBRE? UN ANÁLISIS MULTISITUADO DE LA HISTORIA DE UN CURRÍCULUM NACIONAL EN LA EDUCACIÓN INFANTIL DANESA
}

\section{A Curriculum of Free Play? A Multi-sited Analysis of the History of a National Curriculum in Danish Early Childhood Education}

\author{
Maja PLuM \\ Universidad de Copenhage \\ Correo-e:mplum@hum.ku.dk
}

Recibido: I2 de octubre de 2020. Envío a informantes: 2I de octubre de 2020.

Aceptación definitiva: 9 de abril de 202I

RESUMEN: En 2004 se aprobó el primer currículum en el ámbito de la educación de la primera infancia (ECE) en Dinamarca. Quienes se han opuesto al currículum han argumentado que introduce el pensamiento escolar en el área de la ECE, orientándolo hacia objetivos particulares. Por el contrario, los defensores sostienen que el currículum se refiere al niño natural y debe considerarse como una continuación de una tradición centrada en el niño. Como tal, gran parte del debate parece reflejar un interés en las intenciones de los actores que están detrás del currículum y si la filosofía de estos actores está de acuerdo con una filosofía del pasado centrada en el niño. Una filosofía que a menudo se considera que proviene de la visión de Friedrich Fröbel de un «Jardín de infacia» a principios del siglo XIX y que se inspira más ampliamente en las ideas de un niño natural formuladas por Rousseau en el siglo XVIII.

Con la ambición de historizar el presente, argumentaré que este primer currículum nacional crea el andamiaje de formas de pensamiento que cruzan las dicotomías filosóficas tradicionales entre el interior (naturaleza) y el exterior (objetivo). Por lo tanto, la cuestión histórica no es si este currículum está de acuerdo con una tradición que abarca al niño natural, sino cómo se articula el pensamiento sobre la naturaleza infantil -y las formas en que debe ser redimida- en la obra de Fröbel y sus seguidores daneses Hedvig Bagger y Anna Wulff. Además, el documento 
aborda la forma en que esta noción de naturaleza se transforma en y a través de las psicologías del desarrollo de principios del siglo xx y las teorías sociológicas de la infancia que florecieron a finales del siglo xx y principios del XXI. Esto implica que no me concentro en la filosofía como filosofía, como si fuera coherente y posible de esencializar. En lugar de examinar las intenciones o el significado de los grandes pensadores del pasado de la infancia, me detendré en las formas mismas de pensar la relación entre el conocimiento y el Gobierno que se expone en y a través de la labor de dichos pensadores, pero que también se aplica en ámbitos distintos de la educación, como la administración y la gestión públicas. Así pues, desentrañar la historia de este primer currículum nacional implica referirse a múltiples sitios diferentes.

Palabras Clave: educación y cuidado de la Primera Infancia (ECEC en inglés); currículum; juego; Fröbel; Dinamarca.

AвSTRACT: In 2004 the first national curriculum was passed in the area of early childhood education (ECE) in Denmark. Opponents to the curriculum have argued that it introduces school-like thinking in the area of ECE, making it oriented towards particular goals. On the contrary, proponents argue that the curriculum embraces the natural child and should be seen as a continuation of a child centered tradition. As such, much of the discussion seems to reflect an interest in the intentions of the actors behind the curriculum and whether the philosophy of these actors is in accordance with a child centered philosophy of the past. A philosophy often seen as stemming from Friedrich Fröbel's vision of a Kindergarten in the early $19^{\text {th }}$ century and more broadly drawing on the ideas of a natural child formulated by Rousseau in the $18^{\text {th }}$ century.

With an ambition to historicize the present, I will argue that this first national curriculum scaffolds ways of thinking that crosses traditional philosophical dichotomies between inner (nature) and outer (goal). The historical question therefore is not whether this curriculum is in accordance with a tradition embracing the natural child, but how thinking about child nature - and the ways in which it is to be redeemed - is articulated in the work of Fröbel and his Danish followers Hedvig Bagger and Anna Wulff. Moreover, the paper addresses how this notion of nature is transformed in and through the developmental psychologies of the early $20^{\text {th }}$ century and the sociological theories of childhood blooming in the late $20^{\text {th }}$ and early $2 \mathrm{I}^{\text {st }}$ century. This implies that I do not focus on philosophy as a philosophy - as if coherent and possible to essentialize. Rather than looking into the intentions or meaning of the past great thinkers of kindergarten, I will dwell on the very ways of thinking the relation between knowledge and governing which is exposed in and through the work of such thinkers, but which is also at work in fields distinct from education, such as public administration and management. Thus, unraveling the history of this first national curriculum implies multiple different sites.

KeY words: Early Childhood Education and Care (ECEC); Curriculum; Play; Fröbel; Denmark. 


\section{Introducción}

$\mathrm{E}$ N 2004 SE APROBÓ el primer currículum nacional en el área de la educación y el cuidado infantil (ECEC, según las siglas del nombre del área en inglés) en Dinamarca'. El currículum fue aprobado como una ley que introducía los denominados seis temas de aprendizaje: el desarrollo personal, la competencia social, las habilidades lingüísticas, la competencia del cuerpo y el movimiento, la naturaleza y la ciencia, y la expresión cultural y los valores culturales. El currículum está articulado en forma de temas, más que de asignaturas, los cuales no cubren únicamente áreas de conocimiento que el niño necesita poseer, sino también áreas que reflejan los puntos de encuentro entre el mundo y el desarrollo natural del niño (The Danish Parliament, 2004). Algo que resulta central en este primer currículum nacional es que cada centro infantil debe especificar objetivos claros dentro de los seis temas de aprendizaje, describir sus métodos y documentar y evaluar sus resultados. Los opositores al currículum han argumentado que la ley es un acto de gobierno que no solo reduce la autonomía de la maestra del jardín de infancia, sino también la libertad del niño. A medida que se formulaba la ley (E. M. Andersen, 2004; Ellegaard y Stanek, 2004), se alegó que, en lugar de ser un jardín de juego, el área del ECEC sufriría una transformación hacia la escolarización. Por el contrario, los partidarios de la ley han argumentado que este currículum abraza al niño natural y que debería ser visto como una continuación de una tradición centrada en el niño, que ha sido presumida como fundamental en los países nórdicos (Broström, 2004).

De esta manera, gran parte de la discusión a favor y en contra del primer currículum nacional parece reflexionar sobre el interés que sustenta las intenciones de aquellos que podrían ser denominados los principales actores detrás de la ECEC, en general, y del currículum, en particular: ¿es la intención del Gobierno administrar el área más de cerca? ¿Pretenden limitar la libertad de los niños, fusionando sus años preescolares con sus años escolares? ¿Qué tipo de filosofía se puede interpretar a partir de esta ley? ¿Complementa esta filosofía a aquella del pasado centrada en el niño? Una filosofía que, aunque se afirma que es particularmente nórdica, es vista como proveniente de otros grandes actores (alemanes y suizos) del pasado, a saber, la visión de Friedrich Fröbel sobre un jardín de infantes a principios del siglo XIX (Fröbel, I980) y, en términos más generales, las ideas de un niño natural formuladas por Rousseau en el siglo XVIII (Rousseau, I962).

En este capítulo, me centro menos en los actores y en sus intenciones que en la manera en la que formas particulares de pensar sobre la «naturaleza infantil» -y los modos en que se redime- se articulan y circulan a través de los tiempos.

En Dinamarca, casi el $97 \%$ de los niños de uno a seis años asisten a escuelas infantiles (jardines de infancia y guarderías). Ni los jardines de infancia ni las guarderías son obligatorios como la escuela primaria, que comienza cuando el niño tiene seis años. Las escuelas infantiles están financiadas con fondos públicos y aportadas por maestros de guardería con una licenciatura de una escuela de formación del profesorado y de asistentes que, generalmente, no tienen calificaciones formales. 


\section{¿UN CURRÍCULUM ACERCA DEL JUEGO LIBRE? UN ANÁLISIS MULTISITUADO DE LA HISTORIA DE UN CURÍCULUM NACIONAL EN LA EDUCACIÓN INFANTIL DANESA \\ MAJA PLUM}

Popkewitz (2008, 20II, 20I4) utiliza la noción de «sistemas de razón» para resaltar un interés en la epistemología social de un fenómeno como la educación. Es decir, en lugar de explorar o aclarar cómo se adquirió más conocimiento sobre los niños o cómo evolucionaron las escuelas infantiles, me concentro en las formas en las que el niño se transformó en un sujeto a ser conocido y gobernado de modos particulares. Por lo tanto, utilizo nociones como las de ideas, óptica, lógica y figuras para subrayar analíticamente que no estoy ocupada con la ontología del niño, sino sobre cómo se producen las epistemologías, atribuyendo significado y proporcionando formas de entender «naturalmente» qué es un niño (Baker, 200I). Esto también implica que leo las palabras de pensadores pasados no en una búsqueda hermenéutica de significado, sino como monumentos (Foucault, 1972, p. 7), exponiendo formas particulares de pensar sobre el «jugar», el «niño» y el «jardín de infancia». En lugar de intentar contar una cronología de la institución del jardín de infancia, me sumerjo en momentos de tiempo particulares en los que se puede encontrar esa revelación. Estos momentos son importantes no porque se pusieran ladrillos o porque los actores se pusieran en movimiento, sino porque estos momentos dan inteligibilidad al presente (Foucault, 1977, 200Ib). Esto implica que hay tendencias intelectuales, como la asimilación de Maria Montessori o la difusión de la psicología soviética, que otros verían como aspectos históricos centrales. No lo estoy negando, pero me hallo buscando específicamente momentos en los que surjan rupturas en la forma de pensar sobre el niño-juego-jardín-de-infancia y que tracen líneas al presente.

En el artículo, argumentaré que tanto los opositores como los defensores del currículum de 2004 están en lo cierto. El primer currículum nacional es un acto regulador de gobierno, que dirige al niño, y al área como tal, en una dirección particular; pero no lo hace en contradicción con las formas anteriores (liberadas) de ser un niño o de ser una maestra de jardín de infancia. Al analizar los sistemas de razonamiento que operan en y a través de las teorías y prácticas que forman la historia de este campo en Dinamarca, argumentaré que la idea de redimir a la naturaleza infantil a través del juego (fundamental para el pensamiento de Fröbel) se articula, de hecho, como un acto de liberación. Sin embargo, como tal, también es una forma de gobernar cómo se actúa como un niño que juega y, además, sobre cómo planificar y alimentar este acto de jugar como maestra de jardín de infancia. Por lo tanto, el artículo aborda cómo la «naturaleza infantil» se ha convertido en un objeto curricular que ser facilita a través del juego (mucho antes de que las iniciativas de un currículum nacional estuviesen en proceso). Analiza cómo las nociones de naturaleza y juego infantiles han sido reproducidas y transformadas simultáneamente a través de las psicologías del desarrollo del siglo xx y las teorías sociológicas de finales del siglo xx y principios del XxI sobre el florecimiento infantil. Esto implica que no me enfoco en la filosofía como una filosofía coherente que es posible condensar. En lugar de examinar las intenciones o los significados de los grandes pensadores, del pasado y del presente, sobre los «jardines de infancia», me detendré en los sistemas de razón expuestos en y a través del trabajo de dichos pensadores. Sistemas de razón que también están en funcionamiento 
en campos distintos a la educación, como la administración y la gestión públicas. Por lo tanto, lo multisituado debe entenderse como una forma de mirar más allá de las fronteras que generalmente dibujan los contornos de la ECEC.

\section{El juego curricular}

Aunque se dice que la propia idea de «infancia» ha surgido durante el siglo XVII (Ariès, 1962), el «niño», como una categoría en sí misma -algo que es posible analizar, sobre lo que generar conocimiento y abordar en el gobierno de una población-, es un fenómeno más reciente (Nadesan, 20IO). El siglo XX es, en palabras de Ellen Key (1902), visto, a menudo, como «el siglo del niño». Es una era en la que surgieron nuevas preocupaciones, ciencias e instituciones para ayudar, atender y cuidar al niño. Como tal, la infancia puede ser vista como un espacio imaginado que implica una diferenciación entre edades. En los siglos XVII y XVIII se convirtió en un espacio cada vez más importante para la creación del ciudadano moderno, en el que comenzaron a surgir nuevos expertos y disciplinas, como la psicología y la pedagogía (Bloch, 2006).

El trabajo de Friedrich Fröbel se remonta a principios del siglo xIx y refleja el surgimiento de una preocupación por los miembros más jóvenes de la sociedad. Oponiéndose a las ideas religiosas anteriores del pecado original como incrustado en la naturaleza infantil, Fröbel articula al niño como divino, animado por Dios.

En el nacimiento de un niño humano, lo invisible se vuelve visible, la eternidad se vuelve temporal, lo celestial se vuelve terrenal, el espíritu se vuelve corporal, lo divino se vuelve humano, lo inmanente viene a ser contemplado. Viene a la existencia. (Fröbel, 1980, p35)

Por lo tanto, esa ley eterna de Dios, que funciona sobre todos los asuntos, posee un lugar especial de inmanencia en la carne del niño. Como expone Fröbel, su presencia no es únicamente anunciada en el «exterior, en la naturaleza», sino también en el «interior» del niño (Fröbel, en Sørensen, 1966, p. 63). Esto significa que la observación cuidadosa del niño revelará el espíritu reprimido incrustado en él, haciendo así posible allanar el camino al «impulso de actividad» del niño, a través del cual este espíritu se hace oír (Fröbel, 1980, p. 38). La naturaleza infantil es, como tal, un asunto espiritual que, sin embargo, exige acciones terrenales para ser redimida. La tarea central de esta redención es un asunto de entablar este impulso de actividad (y por lo tanto el espíritu) en relación con la ley eterna incrustada en la naturaleza. Sobre esa base, Fröbel desarrolló los llamados dones de juego, a través de los cuales la eternidad de las formas naturales y la forma natural del niño podrían relacionarse entre sí. Por ejemplo, se ve en la pelota como un juguete que en los escritos de Fröbel constituye un don de juego que incorpora la forma eterna del círculo que también se hace presente en la brecha entre las manos unidas del niño cuando intenta abrazar la pelota (ibid., pp. 43 y ss.). De 
esta manera, en esta óptica, el juego es, en cierto modo, la labor natural del niño -un canal de su espiritualidad interior-, que lo transporta en concordancia con las leyes eternas de la naturaleza. Sin embargo, adoptando esta forma, el juego también se convierte en algo completamente distinto a las ideas de la ociosidad, el ocio o cualquier negocio poco serio alejado de la atención de los adultos. El juego se convierte en una cuestión de suma importancia; y, como tal, algo que debe ser gobernado y prescrito meticulosamente para ser manejado correctamente. Por lo tanto, las ilustraciones y descripciones detalladas acompañaron no solo a la pelota, sino también al cilindro, al rectángulo, a los diferentes juegos de canto, etc. (Fröbel, 1980). Jugar con la pelota tenía una secuencia que hacía que el niño captara primero la forma del círculo y, más tarde, al sustraer la pelota y traerla de vuelta, jugar con ella relacionaba al niño con categorías existenciales como la presencia y la ausencia. Estas prescripciones y el pensamiento de mediar el contenido del mundo a la naturaleza del niño a través de selecciones cuidadosas de la tarea y de la sustancia tienen un sistema de razón que hemos llegado a ver como central en el currículum y en los estudios curriculares, aunque se hallan etiquetados como currículum en los escritos de Fröbel.

Como muestra Hamilton (2009), el propio término currículum está históricamente vinculado a la gestión de las clases dentro de la escolarización: a una cuestión de estructurar un todo dividiéndolo en materias y en secuencia. Es esta idea de la secuencia y de un todo estructurado lo que puede ser encontrado en la lógica de los escritos de Fröbel. Aquí se trata de romper y dividir las leyes eternas del mundo en materias, en el sentido de formas naturales y categorías existenciales que se secuencian, una vez más, en la forma en la que algunos dones de juego se consideran apropiados antes que otros (Fröbel, 1980, cap. 5). Como tal, los escritos de Fröbel exponen un sistema de razón curricular en el que el cuidado infantil es demasiado importante para dejarlo solo a las madres (no importa cuántos consejos haya disponibles sobre la forma «apropiada» de maternidad; Villadsen, 2004, pp. IO2 y ss.). El niño pequeño se convierte en un sitio para inversiones y consejos científicos emergentes, y en un sitio que necesita esfuerzos institucionales. A finales del siglo XIX y principios del xx, esta idea se estableció en Alemania, así como en otros países, en la forma de jardines para niños (jardines de infancia) y de lugares para la formación de las maestras del jardín de infancia (Sørensen, 1966, pp. 60 y ss.).

\section{La fusión de la ciencia natural en Fröbel}

En Dinamarca, estas dos formas institucionales surgieron a finales del siglo XIX. Aunque no fueron la primera pareja en introducir la idea de los jardines de infancia en Dinamarca, la pareja Sophus y Hedevig Bagger fueron una figura central en el sindicato Fröbel danés, así como fundadores de un jardín de infancia y de una institución para educar a las maestras de jardín de infancia (Kristensen y Bayer, 20I5). Con el trabajo de la pareja Bagger, el fröbialismo pierde muchas de 
sus referencias espirituales. Elogiado como un precursor de su tiempo, del «amplio simbolismo» y de la «dependencia del [pensamiento] misterioso» [aclaración añadida], es visto, sin embargo, como atrasado y no en sintonía con el moderno «conocimiento del alma» (Bagger y Bagger, I885, p. 22). Hay, como argumenta Sophus Bagger, una gran diferencia entre la escuela de pensamiento de Fröbel y el actual fröbelianismo danés:

Para nosotros, los ideales de la educación van mucho más allá de lo que lo hicieron para él. Lo que él creía que era posible alcanzar a través del ingenio y de la especulación audaz, lo vemos como algo que solo puede ser alcanzado a través de un largo período de observación exhaustiva de los niños. (S. Bagger, I899, sin numerar)

Esta observación minuciosa de los niños debe ser parcialmente entendida como el conocimiento del alma, o como lo que los Baggers también denominaron psicología. Desde mediados del siglo xviII, la teología había enseñado la «sabiduría del alma y el pensamiento» en los colegios daneses de formación de docentes, y a finales de siglo esta sabiduría se articuló en un objeto explícito de la ciencia, seguido de diferentes publicaciones sobre psicología humana (Bayer y Kristensen, 2015, p. 56). La observación exhaustiva puede entenderse de otra manera, como las observaciones continuas y diarias que las maestras de jardín de infancia debían realizar basándose en el estudio de la psicología, las cuales también enriquecían esta teoría al hacer observaciones de la «disposición, fuerzas, tendencias y colores de temperamento» del niño concreto (H.Bagger, I920, p. 27). Por lo tanto, la circulación danesa del pensamiento fröbeliano le infunde un nuevo tipo de razonamiento no basado en la «especulación», sino en la observación empírica de la naturaleza (infantil).

Esta preocupación por la observación empírica debe ser entendida como parte de una lógica más amplia que celebra los sellos distintivos de las ciencias naturales. El trabajo de Darwin sobre la evolución de las especies se tradujo al danés en I870 y se convirtió en parte de una dispersión más amplia de ideas relacionadas con el progreso humano y social (Bayer y Kristensen, 20I5, p. 43). Lo que otros han denominado la «cuestión social» (Popkewitz, 2006; Villadsen, 2004; Wagner, 200I), resulta central para esta lógica de progreso. Dicha «cuestión social» se entendía como las preocupaciones e inquietudes relacionadas con una población cada vez más urbana y pobre que demuestra lo que era percibido como decadencia física y moral (mendicidad, prostitución, robo, enfermedades, mala higiene, etc.). Por lo tanto, «lo social» se convirtió en un objeto de investigación científica basado en la idea de que funcionaba como un organismo que podía evolucionar hacia etapas superiores de civilización. Sin embargo, la promesa de tal evolución implicaba la investigación analítica de las partes de dicho organismo, de modo que esa intervención pudiera realizarse, imperando así la decadencia de las masas urbanizadas. En 1920, se estableció un departamento nacional danés de ciencias sociales (financiado en parte por la Fundación Rockefeller norteamericana) con el objetivo de reunir conocimiento sobre el ser humano en todos sus componentes, 
promoviendo así el bienestar de la humanidad y la civilización de la sociedad (Buus, 2008). La disciplina emergente de la psicología abarca tales nociones de civilización y el estudio de todos los aspectos de lo humano. El énfasis de los Baggers en la observación minuciosa del niño como tal reproduce un ethos fröbeliano y también lo transforma en una forma moderna en la que la observación del niño está relacionada con la verdad científica emergente del alma del niño; una verdad que implica la idea de progreso social a través de la intervención adecuada sobre la naturaleza infantil.

Esta transformación moderna, sin embargo, no cambió de ninguna manera la lógica del razonamiento de la naturaleza infantil como un sitio de redención. Como narrativa de salvación (Popkewitz, 2008, pp. I8 y ss.), el niño continuó siendo considerado como un nuevo comienzo virgen, que en las manos correctas podría evolucionar hacia la promesa del futuro. Fueron empleadas las actividades curriculares, como los juegos de canto y los dones de juego, aunque se hizo más hincapié en el llamado «juego libre» (Bagger y Bagger, 1885, p. 22) para liberar el impulso de actividad y llevar al niño en contacto con lo que ahora fue articulado como el fenómeno natural del mundo (Bagger, I89I). Se resaltó el punto de vista del niño, teniendo en cuenta que:

A medida que descendemos hacia el niño, entramos en las premisas de sus pensamientos, sentimientos y formas de ver el mundo. Nos permitimos así hacer que el niño nos siga y se beneficie de nuestro cuidado. Sin embargo, a medida que descendemos hacia la visión del niño, no podemos sobrepasar ciertos límites. Ni por un minuto se nos permite olvidar que nos inclinamos hacia él solo para levantarlo a pulso hacia nosotros. (Bagger, 1920, p. 23)

Aunque no es explícito, las reverberaciones espirituales están presentes en esta figura de descender solo para elevar a pulso. Y, como tal, el juego libre es exactamente libre en el sentido de que se pensaba que liberaba el impulso innato de actividad, pero siempre rodeado por la maestra de jardín de infancia que podía guiar cualquier esfuerzo, responder al aliento de preguntas derivadas de la curiosidad del niño y reanudar las actividades libres para proporcionar más instrucciones visuales (Bagger, I89I). Dicho de otra manera, la libertad del niño era dependiente de la cuidadosa observación y orientación de la maestra de jardín de infancia, quien tenía conocimiento del alma del niño. Y el niño que jugaba libremente era un niño que hacía preguntas, se guiaba y se involucraba con la maestra de jardín de infancia.

Esta composición de «libertad» no es nueva, pero traza líneas históricas hacia la división entre lo «interior» y lo «exterior» que es central para la reforma luterana (Baker, 20oI, pp. 335 y ss.). Al no considerar los signos exteriores como aquellos que marcan el camino de Dios en la vida, la orientación interna de uno mismo se convierte en lo que muestra la verdadera creencia. La autonomía o la libertad son una cuestión de la relación interna de uno con Dios. En esta forma de razonamiento, el niño es pensado como vulnerable a diferentes tipos de seducción y, por 
lo tanto, se entiende en necesidad de internalizar la sabiduría de Dios para que sea capaz de establecer una orientación interna. Por lo tanto, la libertad del niño está basada en su dependencia de quienes brindan la orientación a su interior.

Leídos como un monumento, los escritos de la pareja Bagger exponen formas curriculares de razonamiento en las que el «juego libre» se vuelve central. El papel dominante del juego es reproducido, pero es articulado como más «libre» y, como tal, más acorde con la naturaleza infantil. Aunque Fröbel pierde las referencias espirituales explícitas y es fusionado con ideas de las ciencias naturales, la figura de la redención prevalece. El juego libre debe ser entendido como una actividad curricular que relaciona al niño con el mundo a través de la orientación de la maestra; una maestra que, una vez más, es guiada por el conocimiento del alma del niño que se le hace accesible a través de la ciencia emergente de la psicología.

\section{El progreso y la planificación del desarrollo}

Durante la gran expansión del Estado de bienestar, los jardines de infancia se convirtieron en instituciones que no solo se enfocaban en los hijos de las madres de clase trabajadora, que se creían necesitados de vigilancia y ayuda, sino también (en otras instituciones privadas) en los niños de la burguesía, que se creían en necesidad de una mayor estimulación por un par de horas al día. El jardín de infancia floreció en todo el país como un medio para aumentar la igualdad social y asegurar una fuerza laboral para las mujeres (Kristensen y Bayer, 2015). Esto implicó la consolidación de un sindicato de jardines de infancia y la articulación de la profesión del jardín de infancia como una cuyas acciones se encontraban preparadas en función de la base de conocimiento de la psicología del desarrollo (Bayer y Kristensen, 20I5, pp. I20 y ss.). El papel de la psicología del desarrollo, de esta manera, se convierte en fundamental para la idea de la maestra de jardín de infancia como una profesional. En 1967, Knud Rasmussen, profesor de una escuela de formación del profesorado, escribió el libro Psicología del desarrollo, que se convirtió en un superventas y que estaba dirigido a estudiantes de trabajo social y de educación. El libro:

... tiene como objetivo aclarar las características generales del desarrollo, mostrar una secuencia en el progreso del desarrollo y señalar algunas consecuencias relacionadas con las situaciones de influencia en la crianza del niño. (K. Rasmussen, I967, p. II)

Señalar las características generales y esbozar las etapas del desarrollo del niño son, como tal, vistas como clave para establecer el tipo correcto de situaciones de influencia. El niño se conceptualiza como un organismo biológico que se desarrolla en la dinámica existente entre las precondiciones biológicas, que maduran a medida que el niño crece, y las condiciones ambientales de la educación del niño (ibid., p. 13). Así, el conocimiento del alma encuentra su vocabulario en el de las 


\section{¿UN CURRÍCULUM ACERCA DEL JUEGO LIBRE? UN ANÁLISIS MULTISITUADO DE LA HISTORIA DE UN CURÍCULUM NACIONAL EN LA EDUCACIÓN INFANTIL DANESA \\ MAJA PLUM}

ciencias naturales y sociales, en el cual la interacción de causas internas (maduración) y causas externas (situaciones de influencia) es vista como determinante de la personalidad y del desarrollo intelectual del niño. El jardín de infancia es considerado como lleno de «situaciones de influencia», ya que ofrece posibilidades para la autoexpresión "a través del juego, las actividades musicales y el trabajo creativo bajo una guía competente» (ibid., p. I70).

El juego es clasificado y, por lo tanto, hecho posible de identificar a través de las nociones de «juego de funciones», «juego de rol» y «juego de construcción», cada una sirviendo como una ventana para observar la "vida interior del niño» (ibid., p. 207). Mientras circulaban nociones de Anna Freud y Jean Piaget, el niño es visto como egocéntrico en su visión intelectual del mundo, así como lleno de tensiones internas que se hacen oír a través de la autoexpresión. Como tal, el juego se convierte en una actividad vital para el niño y un signo de un desarrollo saludable y natural (ibid., pp. 204 y ss.). A través del juego, el niño no solo practica su capacidad cognitiva desde el primitivismo del pensamiento concreto hacia el de la abstracción; también representa, obra y transforma experiencias y tensiones internas. El «impulso de la actividad» que conecta al niño con el mundo, presente en los escritos de Fröbel y en los de Bagger, ahora se traduce en una «vida interior» (emocional e intelectual) que procesa influencias del mundo exterior, las media y las desarrolla en lo que es repetidamente denominado el «acto creativo» de jugar (ibid., cap. Io). Por lo tanto, el acto libre de jugar ahora no solo sirve únicamente para liberar el impulso de la actividad (liberando el espíritu incrustado en el niño); el juego es un lugar de procesamiento y, como tal, una ventana a las fuerzas de la naturaleza infantil en funcionamiento. "Todas las teorías del propósito están ahora más o menos abandonadas», como afirma Rasmussen (ibid., p. 204), continuando: «y, en cambio, las teorías de la función [...] han adquirido actualidad». Es exactamente esta óptica del juego como algo que tiene una función -ya sea la función del desarrollo cognitivo o la resolución de tensiones internas que conducen al crecimiento personal- lo que lo convierte en una actividad curricular con un carácter central.

En vista de ello, la observación del acto de jugar del niño -y la interacción con el acto- se convierte en una tarea central para la maestra de jardín de infancia. La maestra de jardín de infancia no debe tomar la iniciativa en nombre del niño, sino que siempre debe ser capaz de proporcionar el entorno, los juguetes y los materiales adecuados para permitir la liberación del acto creativo a través del cual el niño se realiza (Rasmussen, 1967, pp. 204, 209). Esto implica la capacidad de identificar la conducta apropiada de juego en relación con la etapa de desarrollo del niño. Por ejemplo, un niño de dos años es -según esta lógica-capaz de realizar un «juego de funciones», colocando dos cosas una encima de la otra o una dentro de la otra, pero jugará y hablará en términos egocéntricos que podrían ser paralelos a otros niños, aunque interactúe con ellos. Es esencial que el jardín de infancia conozca este patrón y no fuerce al niño a -ni pierda la oportunidad de- hacer rimas y ritmos o a contar historias que se correspondan con el niño en esta etapa de desarrollo cognitivo (ibid., p. 206). Como subraya Sutton Smith (1998), esta idea 
de las etapas en el juego de los niños se correlaciona con las nociones evolutivas de progreso en las que el niño progresa y se adapta como un organismo acorde con la evolución de la especie. Proporcionar categorías de juego, en ese sentido, sirve como un mapa de navegación que pronostica lo que vendrá y cómo sentar las bases adecuadas para tal desarrollo.

Esta esperanza, relacionada con la planificación de fundamentos correctos a la luz de una predicción científica, no solo se encuentra en el jardín de infancia, sino que refleja un sistema más amplio de razón incrustado en la expansión del Estado de bienestar. A finales de la década de 1950 y principios de la de 1960, la Administración pública fue problematizada como muy burocrática e incapaz de ver la cohesión de problemas complejos (Andersen, 1995; Rennison, 2004). Por el contrario, la «planificación» se convirtió en la nueva palabra de moda, haciendo hincapié en la necesidad de diseñar lo social para mejorar el progreso de la sociedad. Así, se establecieron grandes comisiones con el propósito de pronosticar y planificar la vida de la población y, como tal, el cumplimiento del Estado de bienestar (Andersen, 1995; Pedersen et al., 1994). Aunque el jardín de infancia, como institución, no entró en la legislación pública hasta 1964 -e incluso entonces no asumió un papel central en los debates e iniciativas políticas (Rasmussen, 2006)-, esta lógica de predicción y planificación para producir la realización del organismo (ya sea la sociedad o el niño) estaba claramente viva en la forma de razonar sobre qué es el niño, el jardín de infancia y el juego. Como una gobernadora profesional del Estado de bienestar, la maestra del jardín de infancia es la que, sobre la base del conocimiento científico (psicología del desarrollo), es capaz de planificar el camino que conduce al desarrollo completo del niño.

\section{El niño competente y su salvación reflexiva}

Mientras que la función de desarrollo del juego podría verse como central para la forma de razonamiento curricular durante los años sesenta y setenta, es exactamente a esta comprensión a la que se presentó oposición durante los años noventa.

... estas tradiciones [influenciadas por la psicología del desarrollo (ed.)] están teñidas de una tendencia a pretender que el juego sea útil -en este caso, una utilidad está relacionada con el desarrollo futuro. (Andersen y Kampmann, 1996, p. 39)

En un libro icónico de 1996, titulado La cultura del juego de los niños, dos profesores de educación, Peter Ø. Andersen y Jan Kampmann, describen, desde una perspectiva más antropológica y sociológica, la actividad de jugar de los niños en dos jardines de infancia en Copenhague. Siguiendo y filmando a los niños durante un período más largo de tiempo, Andersen y Kampmann llegaron a teorizar el juego como procesos de interacción y comunicación a través de los cuales las normas y reglas de conducta son establecidas y constituyen una estructura social 


\section{¿UN CURRÍCULUM ACERCA DEL JUEGO LIBRE? UN ANÁLISIS MULTISITUADO DE LA HISTORIA DE UN CURÍCULUM NACIONAL EN LA EDUCACIÓN INFANTIL DANESA \\ MAJA PLUM}

en el grupo de niños (ibid., cap. 4). Lo que les llamó la atención no fueron tanto las categorías de juego, como el «juego de rol», sino cómo la actividad de jugar involucra diferentes tipos de roles como «el instructor», «el espectador», «el estadista» y cómo los niños retoman una historia, se oponen a ella o la redirigen (ibid., pp. Ior y ss.). Como tal, lo que se vuelve central en esta óptica de juego es la creación continua de significado de los niños, la posición que habitan y su negociación en relación con el significado y el posicionamiento. Esta idea del juego como actividad cultural se basa en las perspectivas de la sociología de la infancia de Brian Sutton-Smith y William Corsaro, y refleja un enfrentamiento más amplio con la psicología del desarrollo que tenía lugar en Dinamarca durante la década de 1990. Por lo tanto, es sintomático que Andersen y Kampmann no solo se opongan a la noción de juego como un medio de desarrollo, sino que emprendan un estudio antropológico relacionado con un lugar y un tiempo particulares, con la ambición de comprender el juego entre niños concretos. Todo esto en oposición a teorizar el juego como una función universal del desarrollo vinculada a la naturaleza infantil per se. De esta manera, dentro de la sociología de la infancia, la óptica del desarrollo se caracteriza por reducir al niño a un objeto de «conversión», en lugar de un sujeto de «ser». Se argumentaba que se debe tener en cuenta el contexto cultural e histórico de la infancia y, como tales, los mundos de la vida de los niños, al igual que se debe ver a los niños como cocreadores activos de su mundo y no solo como receptores pasivos (Corsaro, 1997; James y Prout, 1990).

Sociólogos como Giddens, Beck y Bauman se convirtieron cada vez más en parte del programa de estudios en las escuelas de formación del profesorado que educan a los futuros maestros de jardín de infancia (Bayer y Kristensen, 20I5, p. 170). A diferencia de la comprensión de la naturaleza infantil como un hecho universal, que progresa a través de etapas, el «niño» fue conceptualizado como un fenómeno histórico relacionado con lo que se conoce indistintamente como «tiempos postmodernos», «modernidad radical» y «modernidad tardía» (ibid., pp. 170 y ss.). La sapiencia sobre este nuevo mundo, en el que las normas, los oficios y las tradiciones ya no se transmiten de generación en generación, es percibida como fundamental para las maestras de jardín de infancia porque no pueden confiar en sus propias experiencias de infancia ni en las teorías universales modernas formuladas en un mundo más estable. En cambio, entender al niño particular, en el contexto de un mundo rápidamente cambiante, se considera esencial de acuerdo con esta forma de razonamiento.

Con el telón de fondo de la modernidad tardía, el profesor de psicología Dion Sommer (2002) abrió la puerta a lo que él llama un cambio en los paradigmas, es decir, a un cambio desde la psicología del niño a la psicología infantil. En el centro de la psicología infantil se encuentra una comprensión del desarrollo no como un proceso que tiene lugar únicamente dentro del niño, sino como el resultado de una continua interacción entre el niño, otros niños, adultos y la cultura circundante. En lugar de ser un novicio vulnerable que necesita orientación, el niño es visto como un actor competente nacido con habilidades sociales básicas 
para comunicarse e interactuar ${ }^{2}$, convirtiéndolo, de ese modo, en «un actor en su propio proceso de desarrollo» (ibid., p. 43). Desde esta perspectiva, los jardines de infancia se convierten en arenas en las que el niño interactúa con otros a través de las rutinas diarias y el juego (y participa en tradiciones y normas culturales), desarrollando así su competencia interactiva (ibid., pp. I30 y ss.).

La forma de vida de la familia moderna tardía en nuestra esfera cultural ha resultado en una notable expansión del horizonte social del niño. Esto ha aumentado las demandas sobre el niño en términos de lo que se puede llamar la "competencia interactiva». Esta es la capacidad de leer diferentes situaciones sociales y -a un tiempo y al mismo tiempo- participar activamente mientras se está en sintonía, y también se adapta para ajustarse a muchos contextos sociales diferentes. (ibid., p. II6)

De esta forma, la presuposición de un nuevo tiempo (modernidad tardía) no solo sirve como un análisis sociológico a través del cual se disuelve al niño como organismo universal; también sirve como una descripción de una condición que produce «mayores demandas». La actividad de jugar se vuelve central a este respecto, porque la capacidad de ajustarse o adaptarse simultáneamente en relación con las normas y reglas dadas mientras se mantienen la motivación y el deseo personales es vista como central en la dinámica del juego; por ejemplo, al querer ser parte del juego de «jugar a los autobuses» e inventar el papel de un revisor después de ser rechazado como conductor (ibid., pp. I29 y ss.). Por lo tanto, el juego es destacado como una actividad de negociación y creación de significado continua que integra al niño en narrativas y normas culturales preexistentes, pero que existe simultáneamente como un santuario en el que los niños reinterpretan y transforman su entorno cultural.

En esta forma de razonamiento, el juego constituye una «práctica de convivencia infantil» cotidiana (ibid., p. I3I), a través de la cual el niño ensaya su competencia interactiva fundamental, convirtiéndose así en un niño competente moderno tardío. Como tal, el cambio paradigmático puede ser visto como una transformación en las disciplinas a través de las cuales se ve e interpreta al niño (de la psicología universal a la psicología del contexto inspirada sociológicamente), pero esto también implica una reproducción del «impulso de actividad» o de la «vida interna» presentes a principios de 1900 y 1960. La motivación y el deseo que se considera que el niño pone en juego son vistos, por lo tanto, como una fuente de su capacidad transformadora. Son la motivación personal y el deseo lo que -sintonizado y adaptado- entra en la creación de significado y la negociación del juego. O podríamos decir que el «acto creativo» psicológico del juego se traduce en un acto cultural de «competencia interactiva».

2 Esto refleja, nuevamente, un cambio más amplio dentro de las teorías del apego. Daniel Stern (200o) asume el papel de una figura prominente aquí, argumentando que los niños no nacen en una relación simbiótica de omnipotencia con su madre, como se afirma en las teorías de Bowlby, Mahler y Winnecot. En su lugar, la comunicación social puede ser detectada muy tempranamente, lo que hace que Stern sugiera una sensación temprana de un ser infantil. 


\section{¿UN CURRÍCULUM ACERCA DEL JUEGO LIBRE? UN ANÁLISIS MULTISITUADO DE LA HISTORIA I34 DE UN CURÍCULUM NACIONAL EN LA EDUCACIÓN INFANTIL DANESA \\ MAJA PLUM}

Del mismo modo, la figura de la salvación parece tener éxito en el niño competente. Las nuevas demandas de la modernidad tardía conllevan, de este modo, el riesgo de que no todos los niños se conviertan en el niño popular con un alto «estatus en el juego» (ibid., p. 134). Para convertirse en el niño motivado, en sintonía y flexible, se requiere, en esta forma de razonamiento, el mismo tipo de flexibilidad de la maestra de jardín de infancia. Por un lado, se requiere que sea empática y que reconozca al niño como competente. Por otro lado, nunca debe asignar la responsabilidad del desarrollo al niño, sino que debe articular continuamente las expectativas y aclarar la gramática social de las rutinas culturales y de las situaciones sociales (ibid., pp. 48 y ss., II6 y ss.). Esto implica que su papel como predictora, planificadora y observadora del desarrollo infantil se erosiona y, en su lugar, debe reflexionar repetidamente sobre el tipo de resolución de problemas, las rutinas y las tareas a las que invita al niño: sobre qué aprenderá el niño de ellas y sobre cómo organizar tales rutinas y tareas (ibid., pp. I22 y ss.). Como una forma de razonamiento curricular, la idea analítica de descomponer la sustancia y la secuencia ahora parece funcionar menos como un modo de planificación, y más como una actividad retrospectiva de «reflexión» $\mathrm{y}$ "conciencia» (Andersen y Kampmann, 1996, pp. I83 y ss.; Sommer, 2002). Es decir, observar al niño concreto en ese contexto particular y pensar en qué tipo de competencia interactiva podría mejorarse y qué tipo de tareas y juegos serían apropiados a ese respecto.

Paradójicamente, es este reconocimiento de la competencia del niño y de su desapego de las nociones de «conversión» lo que lo convierte en un sitio de administración cercana: como no hay esquemas universales de desarrollo en los que confiar, cada niño debe ser explorado continuamente. Por lo tanto, la oposición a las ideas del universalismo y a las etapas de progreso no solo dominó en la educación, sino que fue central para el cambio de la Administración pública que tuvo lugar en los años ochenta y noventa en Dinamarca. A diferencia de la planificación centralizada, la descentralización y la delegación de competencias son vistas como medios para fomentar la motivación y la flexibilidad dentro de la institución local, a fin de satisfacer las necesidades de los consumidores (ciudadanos) (Andersen, 1995; Greve, 2007; Rennison, 2004). Sin embargo, como una forma de gobernar este desarrollo local, se exige el acto reflexivo de evaluación. Por lo tanto, aunque se abandonan los grandes planes y las ideas universales de progreso, el acto administrativo de gobernar aún se aferra a la esperanza del «desarrollo de calidad» local, asegurado a través de bucles de retroalimentación, en los que cada institución se hace continuamente consciente de su visión, objetivos y métodos y evalúa su iniciativa desde este punto de vista (Dahler-Larsen, 2004; Vedung, 2006). Es exactamente este acto administrativo de gobernar el que se presenta en la forma curricular de pensar la salvación del niño competente. La erosión del niño universal y abstracto hace imposible que la actividad pedagógica se base en un plan general de «desarrollo normal». Más bien, cada niño debe ser analizado constante y consistentemente en relación con todos los posibles aspectos de sus competencias. Es decir, no hay un mapa general para la navegación. En cambio, el niño competente debe ser salvado (o facilitado) a través de esfuerzos analíticos 
consistentes, desglosando, documentando y evaluando su interacción e interior (Plum, 20I2). En esta forma de razonamiento curricular, la maestra de jardín de infancia se convierte en la gobernadora de un desarrollo local de calidad.

\section{Conclusión: el gobierno curricular del juego}

2004 fue el año en que se aprobó el primer currículum nacional en el área de la educación y el cuidado infantil en Dinamarca. Sin embargo, como se muestra en el análisis anterior, las formas curriculares de razonamiento no resultaban ajenas para el jardín de infancia con anterioridad a la introducción de este currículum. Por lo tanto, los defensores del currículum tienen razón cuando dicen que este está en línea con el enfoque del pasado, centrado en el niño. La óptica de juego fröbeliana, como una liberación del espíritu reprimido del niño, todavía figura en la idea del juego libre como un acto creativo que expone los procesos emocionales y cognitivos del niño. Y está presente en la óptica del juego como una cuestión de negociación y creación de significado del niño competente. Para liberar al espíritu, la vida interior o el niño competente de la modernidad tardía implican la guía de una maestra de jardín de infancia que es capaz de observar, guiar, planificar o facilitar analíticamente al «niño natural»; un niño cuyo interior natural -y, como tal, su forma de ser conocido y gobernado- está cambiando a través del tiempo.

Los opositores al currículum, por lo tanto, también tienen razón cuando afirman que la ley es un acto incrementado de gobernanza. Es una forma de querer saber más sobre el niño en su especificidad local. En cierto modo, esto reduce la autonomía de la maestra del jardín de infancia; pero no porque esta viviera en una era de profesionalismo libre. Más bien, porque la razón científica de la psicología del desarrollo solía darle derecho a planificar el progreso del niño natural universal. La erosión de las ideas del progreso universal ahora transforma su profesionalismo en una cuestión de constante desarrollo de la calidad. Debe documentar y evaluar continuamente al niño competente particular en su contexto local.

Como se mostró con mayor profundidad anteriormente (Plum, 20I4, 20I8), el razonamiento del niño competente, de esta manera, parece correlacionarse con relativa facilidad con las preocupaciones y la administración moderna relacionadas con PISA. A pesar de que la filosofía de la sociología de la infancia, y su insistencia en el niño como un «ser», puede ser vista como a kilómetros de distancia respecto a la comprensión dominante de los niños en una era de rendición de cuentas, el niño competente se traduce con poca fricción en una reserva de futuro capital humano.

\section{Bibliografía}

Andersen, E. M.: «Læreplaner; glem ikke barnet» [«El currículum en la guardería; no hay que olvidar al niño»], Børn \& Unge, I3 (2004). 


\section{6 \\ ¿UN CURRÍCULUM ACERCA DEL JUEGO LIBRE? UN ANÁLISIS MULTISITUADO DE LA HISTORIA DE UN CURÍCULUM NACIONAL EN LA EDUCACIÓN INFANTIL DANESA \\ MAJA PLUM}

ANDERSEN, N. Å.: Selvskabt forvaltning. Forvaltningspolitikkens og centralforvaltningens udvikling i Danmark 1900-1994. [La administración becha a si misma. El desarrollo de la politica de la administración y la administración central en Dinamarca 1900-1994], Copenhague, Nyt fra Samfundsvidenskaberne, 1995.

Andersen, P. Ø. y Kampmann, J.: Børns legekultur [Cultura lúdica infantil], Viborg, Munksgaard Rosinante, 1996.

ÀrIES, P.: Centuries of childhood, Londres, Cape, 1962.

BAgger, H.: Den danske Børnehave, Haandbog i den første Undervisning for Hjemmet, Børnehaven, Asylet og Smaaskolen [El jardín de infancia danés: Manual de la primera educación, para el hogar, el jardín de infancia, el asilo y la escuela pequeña], Copenhague, N. C. Roms Forlagsforretning, I89I.

BAGGER, H.: Smaabørnslererinden i Hjemmet, asylet og Folkebørnehaven [El maestro de jardin de infancia en el hogar, el asilo y el jardín de infancia público], Copenhague, N.C. Roms Forlagsforretning, 1920.

Bagger, H. y Bagger, S.: Den danske Børnehave, Bemerkninger om smaa Børns Opdragelse og Undervisning [El jardín de infancia danés: observaciones sobre la educación y la enseñanza de los niños pequeños], Copenhague, Marius Møller, I885.

BAGGER, S.: «Børnehaven, frøbelsk eller ikke Frøbelsk» [«El jardín de infancia: fröbliano o no fröbliano»], en LAngsted, A. (ed.): Nutids Børneopdragelse og Skolegang. Orienterende Betragtninger [Crianza y escolarización de los niños modernos. Consideraciones sobre su orientación], Copenhague, H. Hagerups Boghandel, 1899.

BAKER, B. M.: In perpetual motion: theories of power, educational history and the child, Nueva York y Oxford, Peter Lang, 20oI.

Bayer, S. y Kristensen, J. E. (eds.): Kald og Kundskab [Llamada o vocación], Copenhague, Upress, 20I5.

BlOCH, M. N.: «Educational theories and pedagogies as technologies of power/knowledge: Educating the young child as a citizen of an imagined nation and world», en BLOCH, M. N.; Kennedy, D.; Lightfoot, T. y Weyenberg, D. (eds.): The child in the world/the world in the child. Education and the configuration of a universal, modern and globalized childhood, Nueva York, Palgrave Macmillan, 2006, pp. 2I-42.

Broström, S. (ed.): Pedagogiske lareplaner - at arbejde med didaktik i børnehaven [El curriculum en la guardería - para trabajar la didáctica en el jardín de infancia], Aarhus, Systime, 2004 .

BuUs, H.: Indretning og efterretning. Rockefeller Foundations indflydelse på den danske velfardsstat 1920-1970 [Organizar y tomar nota. La influencia de la Fundación Rockefeller en el Estado de bienestar danés 1920-1970], Copenhague, Museum Tusculanums Forlag, 2008.

Corsaro, W. A.: The sociology of childhood, Thousand Oaks, Calif. y Londres, Pine Forge Press, 1997.

Dahler-Larsen, P.: Evaluering og magt [Evaluación y poder], Aarhus University, 2004.

EllegaARd, T. y STANeK, A. H. (eds.): Lareplaner i børnehaven. Baggrund og perspektiver [El plan de estudios en el jardín de infancia. Antecedentes y perspectivas], Vejle, Kroghs Forlag, 2004 .

Foucault, M.: The Archaeology of Knowledge, Londres, Routledge, 1972. (Trad. castellana: La arqueología del saber, México, Siglo XXI, 1978).

Foucault, M.: Discipline and Punishment. The Birth of the Prison, Londres, Penguin Books, 1977. (Trad. castellana: Vigilar y castigar: el nacimiento de la prisión, México, Siglo XXI, I976).

Foucault, M.: «Nietzsche - geneologien, historien» [«Nietzsche - la genealogía, la historia»], en Foucault, M. (ed.): Talens forfatning [La constitución de la palabra], Copenhague, Hans Reitzels Forlag, 200I. 
¿UN CURRÍCULUM ACERCA DEL JUEGO LIBRE? UN ANÁLISIS MULTISITUADO DE LA HISTORIA DE UN CURÍCULUM NACIONAL EN LA EDUCACIÓN INFANTIL DANESA MAJA PLUM

FröBeL, F.: Småbørnspredagogik [Educación infantil], Copenhague, Nyt Nordisk Forlag, I980. Greve, C. (Ed.): Offentlig ledelse og styring [Administración y gestión pública], Copenhague: Jurist- og Økonomforbundets Forlag, 2007.

Hamilton, D.: «On the origins of the educational terms class and curriculum», en BAKer, B. (ed.): New Curriculum History, Rotterdam, Sense, 2009. (Trad. castellana: «Origen de los términos clase y currículum», Revista de Educación, 295 [1991], pp. 187-205).

James, A. y PROUT, A.: Constructing and reconstructing childhood: contemporary issues in the sociological study of childhood, Londres, Falmer, I990.

Key, E.: Barnets Aarbundrede [El siglo del niño], Copenhague, 1902. (Trad. castellana: El siglo de los niños, Buenos Aires, Albatros, 1945).

Kristensen, J. E. y BAyer, S. (eds.): Kamp og Status. De lange linjer i børnehaveinstitutionens og pedagogprofessionens historie 1820-20I5 [Lucha y estatus. Dibujando las largas líneas de la historia de las guarderías y de la profesión de maestro de párvulos, 1820-2015], Copenhague, Upress, 2015.

NADESAN, M. H.: Governing childhood into the 2Ist century: biopolitical technologies of childhood management and education, Basingstoke, Palgrave Macmillan, 2010.

Pedersen, O. K.; Bjerregaard, R.; Elming, P.; Hansen, S.; Hummelmose, H.; Højland, P. y LaRsen, J.: Demokratiets lette tilstand. Syv beslutningstagere om Danmark og fremtiden [La frágil condición de la Democracia. Siete politicos sobre Dinamarca y el futuro], Copenhague, Spektrum, I994.

Plum, M.: «The emergence of the analytical method in early childhood education - the scientific effort to produce the learning child for the nation in a global era», International Journal of Qualitative Studies in Education, 25(5) (2012), pp. 645-664.

Plum, M.: "A 'globalised' curriculum - international comparative practices and the preschool child as a site of economic optimisation», Discourse: Studies in the Cultural Politics of Education, 35(4) (2014), pp. 570-583.

Plum, M.: «Humanism, administration and education: The demand of documentation and the production of a new pedagogical desire», en Ball, S. (ed.): Foucault and Education: Putting theory to work, Londres, Routledge, 2018, pp. 166-182.

Popkewitz, T. S.: «The idea of science as planning was not planned», en Hofstetter, B. S. R. (ed.): Passion, fusion, tension. New education and educational sciences, Nueva York, Peter Lang, 2006, pp. I43-167.

PopkewiTZ, T. S.: Cosmopolitanism and the age of school reform: science, education, and making society by making the child, Nueva York, Routledge, 2008. (Trad. castellana: El cosmopolitismo en la era de la reforma escolar. La ciencia, la educación y la construcción de la sociedad mediante la construcción de la infancia, Madrid, Morata, 2009).

PopkewiTz, T. S.: «Curriculum history, schooling and the history of the present», History of Education, 4O(I) (201I), pp. I-I9. doi: I0.1080/0046760X.2010.507222

Popkewitz, T. S.: «Social Epistemology, the reason of 'reason' and the curriculum studies», Education Policy Analysis Archives, 22(22) (20I4). doi:I0.I4507/epaa.v22n22.20I4.

Rasmussen, K.: Udviklingspsykologi. Barndom og pubertet [Psicología del desarrollo. Infancia y pubertad], Copenhague, Gyldendal, 1967.

ReNnison, B. W.: «Ledelsesbegrebets historie i den offentlige sektor» [«La historia del concepto de gobernar en el sector público»], en Pedersen, D. (ed.): Offentlig ledelse i managementstaten [La gobernanza pública en el Estado gestor], Frederiksberg, Samfundslitteratur, 2004, pp. 85-103.

Rousseau, J.-J.: Emile - eller om opdragelsen [Emilio - sobre la educación], Copenhague, Borgens forlag, 1962 .

Sommer, D.: Barndomspsykologi. Udvikling i en forandret verden [Psicología de la infancia. El desarrollo en un mundo cambiado] (2. ${ }^{\text {a }}$ ed.), Copenhague, Hans Reitzel, 2002. 
STERN, D. N.: The interpersonal world of the infant: a view from psychoanalysis and developmental psychology, Nueva York, Basic Books, 2000.

SutTon-Smith, B.: The ambiguity of play, Cambridge, Mass. y Londres, Harvard University Press, 1998.

Sørensen, K. G.: Opdragelsens historie [Historia de la educación] (vol. 3), Copenhague, Gyldendals pædagogiske bibliotek, 1966.

The Danish Parliament: Pedagogiske lereplaner i dagtilbud til børn [El currículum en las guarderías para niños], 2004.

VEDUNG, E.: «Utvärdering som megatrend, gigatrend och fyra böljer» [«Evaluación como megatendencia, gigatendencia y cuatro cambios»], en HANSEN, H. F. (ed.): Den organiserede forvaltning. Politik, viden og vardier $i$ samspil [La administración organizada. Politica, conocimiento y valores entrelazados], Copenhague, Forlaget Politiske Studier, 2006, pp. IOS-I5O.

Villadsen, K.: Det sociale arbejdes genealogi. Om kampen om at gøre fattige og udstødte til frie mennesker [La genealogía del trabajo social. La lucha por convertir a los pobres y a los marginados en seres humanos libres], Copenhague, Hans Reitzels Forlag, 2004.

Wagner, P. A History and Theory of the Social Sciences, Londres, SAGE Publications, $200 I$.

(Traducción al español de Domingo Barroso, Universidad de Granada) 